\title{
DEVELOPING A QUANTUM CIRCUIT SIMULATOR API
}

\author{
MIHAI DORIAN Stancu \\ Ph.D. student, Faculty of Economic Sciences/Cybernetics and Statistics Department, "Lucian Blaga" University \\ of Sibiu, Sibiu, Romania, stancu.mihai.dorian@gmail.com \\ EMIL MARIN Popa \\ Professor, Faculty of Economic Sciences/Cybernetics and Statistics Department, "Lucian Blaga" University of \\ Sibiu, Sibiu, Romania, emilmpopa@yahoo.com
}

\begin{abstract}
In this paper we propose the design and implementation of a quantum circuit simulator API. Currently the API allows users to implement, debug and test the following two quantum algorithms: BernsteinVazirani's algorithm and Simon's Algorithm. The goal is to create a framework that will allow quantum computer scientists to easily develop new quantum algorithms.
\end{abstract}

Key words: quantum mechanics, quantum gate, quantum circuit, quantum algorithm, quantum simulator

\section{Introduction}

Computers have become extremely powerful machines. As of November 2014, according to the Top500 project [1], the fastest known supercomputer is Tianhe-2, in China, having 3,120,000 processor cores and running at 33.86 petaflops/s. Even smartphones today have more processing power than the onboard computers from the Apollo missions to the Moon, in the 1960s. Processing power will continue to grow in the next years, in accordance with Moore's Law [2], as the number of transistors in integrated circuits continues to double every 18 months. Thus transistors will get smaller and smaller, until they reach a minimum level of miniaturization. In 2012, scientists have created a single-atom transistor, approximately $1 \times 10^{-10}$ meters in size [3], but it could only function at very low temperatures. As of September 2014, the smallest commercially available transistor had $1.4 \times 10^{-10}$ meters [4].

At the atomic scale, manufacturing reliable transistors becomes increasingly difficult due to the quantum mechanical effects that must be overcome. A very important question now is whether new devices can be created, that use quantum mechanics, to help push Moore's Law into the future, after the point when transistors, as we know them, reach their minimum size. Can we create quantum mechanical computers?

Richard Feynman was among the first to ask this question [5]. Since then, many researchers have tried and succeeded to implement small quantum computations and different quantum algorithms. Even commercial quantum computers have already been created, such as D-Wave One (2010) and D-Wave Two (2013) [6], although it has not yet been proven whether they exceed the computational capabilities of existing supercomputers or not. Also, there are many open questions regarding the kinds of problems that can be solved more efficiently using quantum computers, and the benefits that quantum computing has to offer over classical computing. This is why quantum computing has become a highly active field of research in recent years.

In the following sections we give a short introduction to classical computing and quantum computing. Then, we present the quantum circuit simulator API that we have developed, and we use it to implement two known quantum algorithms: Bernstein-Vazirani's algorithm and Simon's Algorithm. Finally we present our conclusions and plans for future development.

\section{Classical Computing}

In classical information theory, the smallest unit of information is the bit (binary digit). A bit can be either 0 or 1 . In computer processors, a bit is represented by a voltage, in a hard-disk, it is represented by a magnetic domain, in an optical fiber by light, etc. 
In order to perform operations on bits, processors use transistors. Transistors are electrical devices that behave like switches. If a non-zero voltage is applied, they allow current to pass through them, otherwise not. Transistors can be connected into circuits and grouped together into so-called logic gates, to implement boolean functions that have one or more input bits and one output bit.

Following are a few examples of logic gates, together with their symbols and truth tables [7]:
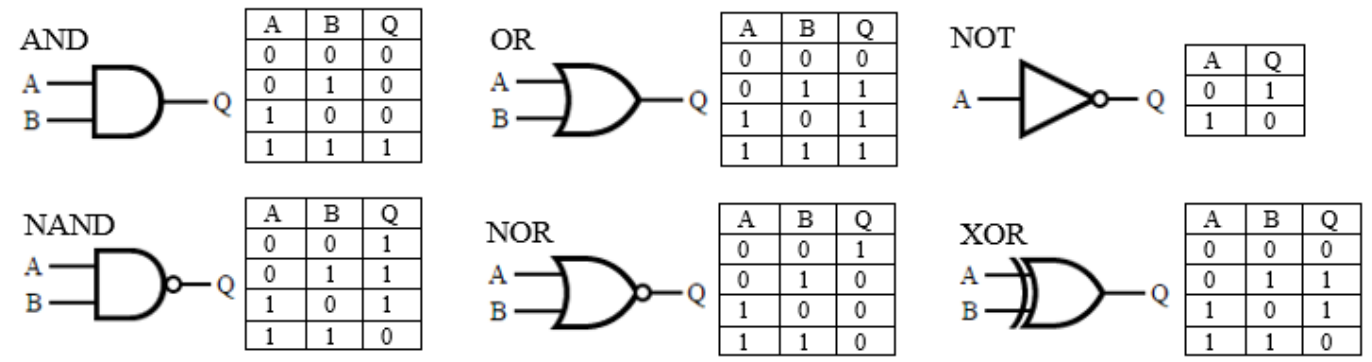

Figure 1: Logic gate examples, together with corresponding symbols and truth tables

In 1913 the American logician Henry M. Sheffer showed that the NAND (NOT + AND) gate is functionally complete [8], or universal, i.e. any logic circuit can be built using just NAND gates. This means that even the most complex boolean function can be implemented using only a sequence of NAND gates.

\section{Quantum Computing}

\subsection{Quantum bits}

In quantum computing, the unit of quantum information is the qubit (quantum bit). A qubit can be represented by the spin of an electron, the spin of a proton, the polarization of a photon, etc.

A qubit is the simplest two-state quantum mechanical system. The two states are called basis states and are represented by the following Dirac or "bra-ket" notation: $|0\rangle,|1\rangle$. When measured, a qubit can be either $|0\rangle$ or $|1\rangle$. Unlike the classical bit, before it is measured, the qubit can be in a linear combination of the basis states, called a superposition [9]:

$|\psi\rangle=\alpha_{0}|0\rangle+\alpha_{1}|1\rangle, \alpha_{0}, \alpha_{1} \in \mathbb{C},\left|\alpha_{0}\right|^{2}+\left|\alpha_{1}\right|^{2}=1$

$\alpha_{0}$ and $\alpha_{1}$ are called probability amplitudes.

We can also use the following matrix notation:

$|\psi\rangle=\left[\begin{array}{l}\alpha_{0} \\ \alpha_{1}\end{array}\right],|0\rangle=\left[\begin{array}{l}1 \\ 0\end{array}\right],|1\rangle=\left[\begin{array}{l}0 \\ 1\end{array}\right]$

A system of two qubits would have $2^{2}=4$ basis states: $|00\rangle,|01\rangle,|10\rangle,|11\rangle$.

A superposition in such a system would be written as:

$|\psi\rangle=\alpha_{00}|00\rangle+\alpha_{01}|01\rangle+\alpha_{10}|10\rangle+\alpha_{11}|11\rangle$

$\left|\alpha_{00}\right|^{2}+\left|\alpha_{01}\right|^{2}+\left|\alpha_{10}\right|^{2}+\left|\alpha_{11}\right|^{2}=1$

Again, we give the matrix notation as well:

$|\psi\rangle=\left[\begin{array}{l}\alpha_{00} \\ \alpha_{01} \\ \alpha_{10} \\ \alpha_{11}\end{array}\right],|00\rangle=\left[\begin{array}{l}1 \\ 0 \\ 0 \\ 0\end{array}\right],|01\rangle=\left[\begin{array}{l}0 \\ 1 \\ 0 \\ 0\end{array}\right],|10\rangle=\left[\begin{array}{l}0 \\ 0 \\ 1 \\ 0\end{array}\right],|11\rangle=\left[\begin{array}{l}0 \\ 0 \\ 0 \\ 1\end{array}\right]$

In general, for $\mathrm{n}$ qubits there are $2^{\mathrm{n}}$ basis states, and a superposition would be written as:

$|\psi\rangle=\alpha_{0 \ldots 00}|0 \ldots 00\rangle+\alpha_{0 . .01}|0 \ldots 01\rangle+\ldots+\alpha_{1 \ldots 11}|1 \ldots 11\rangle$,

$\left|\alpha_{0 \ldots 00}\right|^{2}+\left|\alpha_{0 \ldots 01}\right|^{2}+\cdots+\left|\alpha_{1 \ldots 11}\right|^{2}=1$

In matrix notation we have:

$|\psi\rangle=\left[\begin{array}{c}\alpha_{0} \\ \alpha_{1} \\ \vdots \\ \alpha_{2^{n}-1}\end{array}\right],|0 \ldots 00\rangle=\left[\begin{array}{c}1 \\ 0 \\ \vdots \\ 0\end{array}\right],|0 \ldots 01\rangle=\left[\begin{array}{c}0 \\ 1 \\ \vdots \\ 0\end{array}\right], \ldots,|1 \ldots 11\rangle=\left[\begin{array}{c}0 \\ 0 \\ \vdots \\ 1\end{array}\right]$

We call $|\psi\rangle$ a quantum register. When we measure $|\psi\rangle$, we get $|i\rangle$ with probability $\left|\alpha_{i}\right|^{2}$. The result of the measurement, in general, cannot be predicted. The probabilistic nature of the measurement operation is an inherent characteristic of quantum mechanics [9]. 
If we wish to measure just one out of the $\mathrm{n}$ qubits in the quantum register, the initial superposition collapses to a new superposition and the following occurs: the probability amplitudes of all base states that do not contain the value that was measured for the target qubit become 0 and the quantum register is renormalized [11].

For example, assume we have the following superposition: $\frac{1}{\sqrt{3}}|100\rangle+\frac{1}{\sqrt{3}}|011\rangle+\frac{1}{\sqrt{3}}|010\rangle$. Now assume that we measure the first qubit and we observe it to be $|0\rangle$. The superposition in this case becomes $\frac{1}{\sqrt{2}}|011\rangle+\frac{1}{\sqrt{2}}|010\rangle$

Currently there are two main quantum computing models: one based on quantum circuits and another called adiabatic quantum computing. It has been shown in [10] that the two models are equivalent. In this paper we are only interested in the quantum circuit model, since it is the most similar to the classical computing model, as we will see in the following subsections.

\subsection{Quantum Gates}

A quantum gate is a transformation applied to a quantum register. Unlike most classical logic gates, quantum gates are always reversible, and are represented by unitary matrices. They operate on one or more qubits, just like the classical gates operate on one or more bits.

We list below a few examples of quantum gates, together with their symbolic notation and matrix representations [11]:

Pauli-X

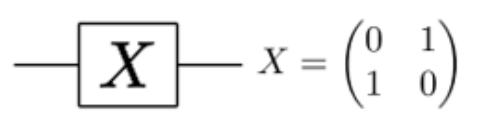

Pauli-Z Hadamard

Phase shift

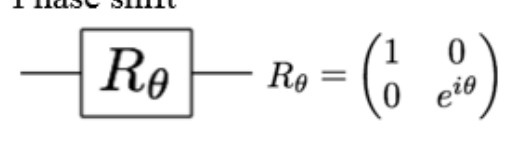

Swap
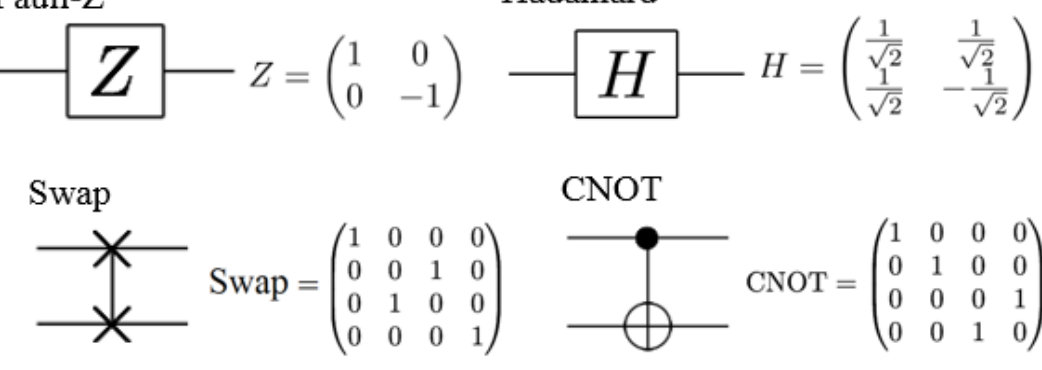

\section{CNOT}

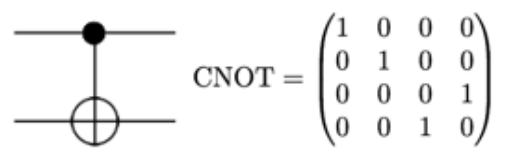

Figure 2: Quantum gate examples, together with corresponding symbols and matrices

For example, in order to apply the Hadamard gate to $|0\rangle$ :

$H|0\rangle=\frac{1}{\sqrt{2}}\left(\begin{array}{cc}1 & 1 \\ 1 & -1\end{array}\right)\left[\begin{array}{l}1 \\ 0\end{array}\right]=\frac{1}{\sqrt{2}}\left[\begin{array}{l}1 \\ 1\end{array}\right]=\frac{1}{\sqrt{2}}\left[\begin{array}{l}1 \\ 0\end{array}\right]+\frac{1}{\sqrt{2}}\left[\begin{array}{l}0 \\ 1\end{array}\right]=\frac{1}{\sqrt{2}}|0\rangle+\frac{1}{\sqrt{2}}|1\rangle$

The CNOT (Controlled-NOT) turns $|00\rangle$ to $|00\rangle,|01\rangle$ to $|01\rangle,|10\rangle$ to $|11\rangle$ and $|11\rangle$ to $|10\rangle$, and thus would turn $\alpha_{00}|00\rangle+\alpha_{01}|01\rangle+\alpha_{10}|10\rangle+\alpha_{11}|11\rangle$ to $\alpha_{00}|00\rangle+\alpha_{01}|01\rangle+\alpha_{10}|11\rangle+\alpha_{11}|10\rangle$.

The measurement operation, even though it is not a quantum gate, also has a symbolic notation:

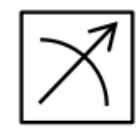

Figure 3: Symbol for quantum measurement

Just as NAND is a universal gate for classical circuits, the Toffoli gate, or CCNOT gate, is a universal gate for quantum circuits [11]:

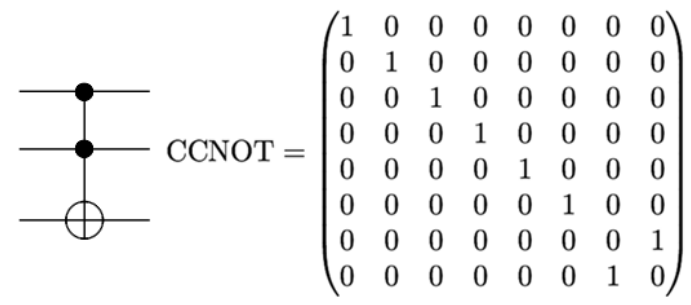

Figure 4: Toffoli (CCNOT) gate symbol and associated matrix 


\subsection{Quantum Circuits}

Quantum gates can be combined to form quantum circuits. The quantum circuits, just like the quantum gates, are reversible and have associated unitary matrices. This means that the number of input qubits must always be equal to the number of output qubits. Thus the matrix associated to any quantum circuit is basically a permutation matrix.

As a concrete example, let us consider the following quantum circuit:

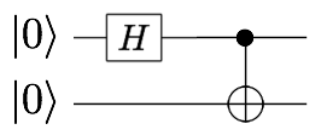

Figure 5: Quantum circuit for obtaining a Bell state

After applying the Hadamard gate, the first qubit becomes $\frac{1}{\sqrt{2}}|0\rangle+\frac{1}{\sqrt{2}}|1\rangle$, and the state of the entire system is $\frac{1}{\sqrt{2}}|00\rangle+\frac{1}{\sqrt{2}}|10\rangle$. Applying a CNOT to $|00\rangle$ results in $|00\rangle$, and applying a CNOT to $|10\rangle$ results in $|11\rangle$. Thus, after applying the CNOT gate, the final state of the system becomes $\frac{1}{\sqrt{2}}|00\rangle+\frac{1}{\sqrt{2}}|11\rangle$. This is called a Bell state.

We could have also calculated $U$ directly: $U=C N O T \times(H \otimes I)$, where $\otimes$ is the tensor product:

$$
\begin{gathered}
U=\left(\begin{array}{llll}
1 & 0 & 0 & 0 \\
0 & 1 & 0 & 0 \\
0 & 0 & 0 & 1 \\
0 & 0 & 1 & 0
\end{array}\right) \times\left(\frac{1}{\sqrt{2}}\left(\begin{array}{cc}
1 & 1 \\
1 & -1
\end{array}\right) \otimes\left(\begin{array}{ll}
1 & 0 \\
0 & 1
\end{array}\right)\right)=\left(\begin{array}{llll}
1 & 0 & 0 & 0 \\
0 & 1 & 0 & 0 \\
0 & 0 & 0 & 1 \\
0 & 0 & 1 & 0
\end{array}\right) \times \frac{1}{\sqrt{2}}\left(\begin{array}{cccc}
1 & 0 & 1 & 0 \\
0 & 1 & 0 & 1 \\
1 & 0 & -1 & 0 \\
0 & 1 & 0 & -1
\end{array}\right) \\
U|00\rangle=U\left[\begin{array}{l}
1 \\
0 \\
0 \\
0
\end{array}\right]=\frac{1}{\sqrt{2}}\left(\begin{array}{cccc}
1 & 0 & 1 & 0 \\
0 & 1 & 0 & 1 \\
0 & 1 & 0 & -1 \\
1 & 0 & -1 & 0
\end{array}\right)\left[\begin{array}{l}
1 \\
0 \\
0 \\
0
\end{array}\right]=\frac{1}{\sqrt{2}}\left[\begin{array}{l}
1 \\
0 \\
0 \\
1
\end{array}\right]=\frac{1}{\sqrt{2}}\left[\begin{array}{l}
1 \\
0 \\
0 \\
0
\end{array}\right]+\frac{1}{\sqrt{2}}\left[\begin{array}{l}
0 \\
0 \\
0 \\
1
\end{array}\right]=\frac{1}{\sqrt{2}}|00\rangle+\frac{1}{\sqrt{2}}|11\rangle
\end{gathered}
$$

\section{Quantum Circuit Simulator}

\subsection{API}

In [12] we see that there have been many quantum circuit simulators, visualizers, quantum programming languages and markup languages developed to date. These have been implemented in various languages, such as C/C++, Java, Mathematica, Matlab, Maple, Python, PHP, Javascript, etc. The main reason we decided to create our own quantum circuit simulator was, first of all, in order to better grasp the quantum algorithms that we chose to implement so far, and to develop an intuition for quantum phenomena.

We decided to implement the simulator in C\#, using the .NET Framework, since we have more experience with these, and also because they offer many benefits, such as: they are object-oriented, they provide reasonable performance and allow rapid application development, with many tools available that help test and debug the code very easily.

Below is a diagram of the most important classes and methods we have implemented so far:

\begin{tabular}{|l|l|}
\hline Register Class & Operation Abstract Class \\
$-\quad$ void Apply(Operation operation, int index) & $-\quad$ Operation TensorProduct(Operation operation) \\
$-\quad$ void Apply(Operation operation) & \\
$-\quad$ void Apply(Func $<$ int, int> function) & Identity Class - inherits Operation \\
$-\quad$ string Measure(int index) & Hadamard Class - inherits Operation \\
\hline
\end{tabular}

The Register class uses internally an array of complex numbers to represent the probability amplitudes, and the Operation class uses internally a 2-dimensional array with complex elements to hold the unitary matrix associated with the transformation. 
As can be seen, the API is extremely simple. To better understand it, in the following sections we will present the quantum circuits for two well-known algorithms, and will implement them using the API that we have developed.

\subsection{Bernstein-Vazirani's Algorithm}

Problem Statement: We are given a function $f:\{0,1\}^{n} \rightarrow\{0,1\}$ as a black box. We know that $f(x)=u \cdot x$ for some "hidden" $u \in\{0,1\}^{n}$. We need to find $u$.

We consider that the function is given to us as the following black box quantum circuit:

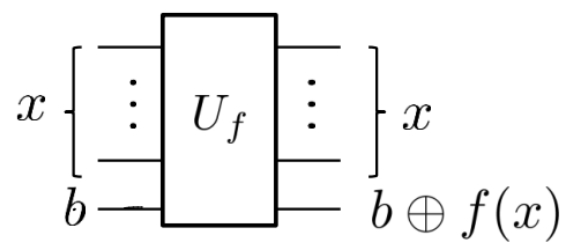

Figure 6. Black box quantum circuit

(b is a control bit and $\oplus$ is the XOR operation)

The quantum circuit that solves this problem is the following:

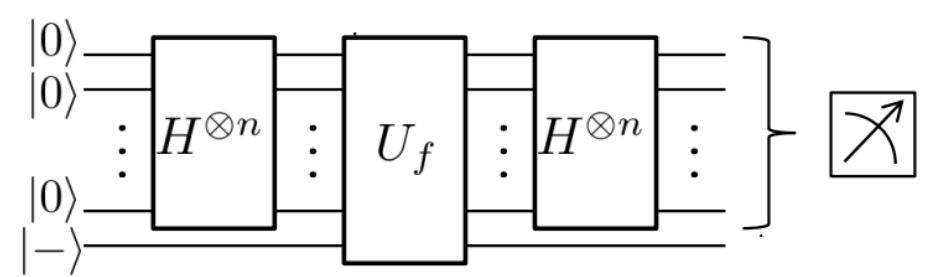

Figure 7. Quantum circuit for Bernstein-Vazirani's algorithm ( $H^{\otimes n}$ is the Hadamard gate tensored $n$ times)

The C\# code that implements the above quantum circuit using our API is the following:

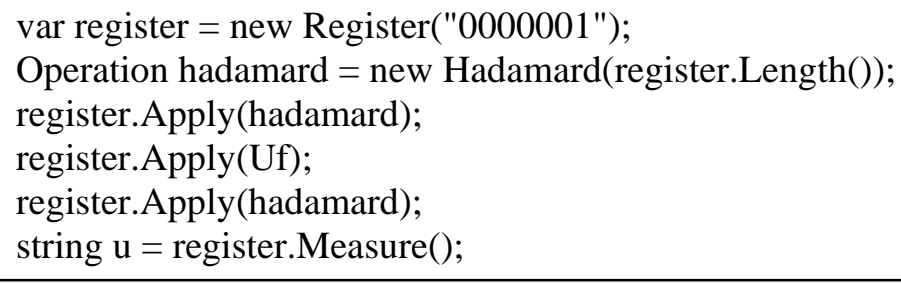

Figure 8. Bernstein-Vazirani's algorithm, implemented in C\#, using our quantum circuit simulator

\subsection{Simon's Algorithm}

(Uf is a user-defined method that satisfies the problem definition)

Problem statement: We are given a function $f:\{0,1\}^{n} \rightarrow\{0,1\}^{n}$ as a black box. We know that there is a secret string $s \in\{0,1\}^{n}$ such that $f(x)=f(x \oplus s)$. We need to find $s$.

We consider that the function is given to us as the following black box quantum circuit:

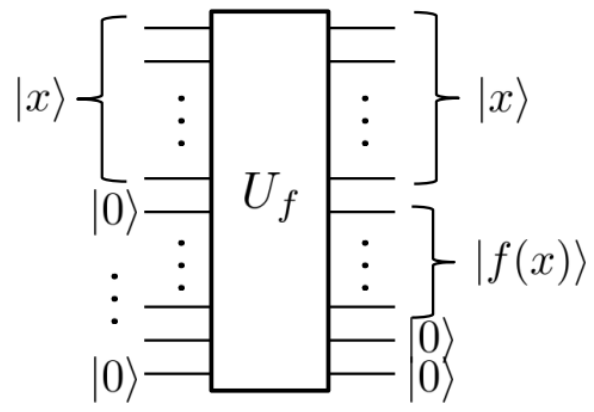

Figure 9. Black box quantum circuit 
The following quantum circuit, repeated several times, helps solve this problem:

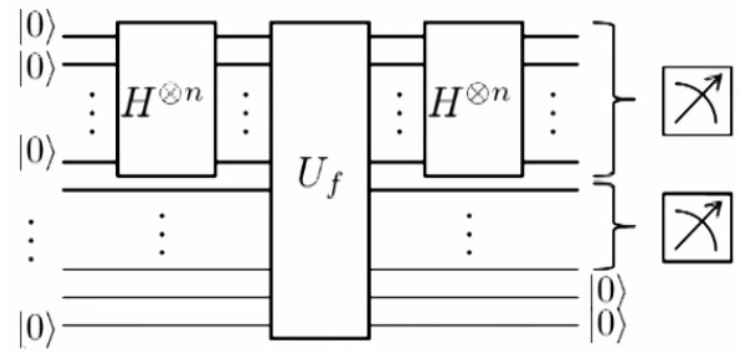

Figure 10. Quantum circuit for Simon's Algorithm

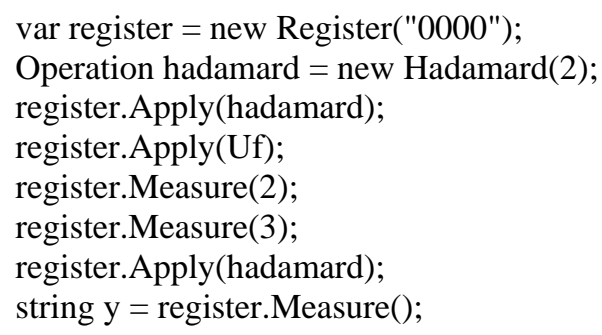

Figure 11. Part of Simon's algorithm, implemented in C\#, using our quantum circuit simulator

\section{Conclusions}

The API we have developed is extremely easy to use. Currently we have only a few classes, since only these were required for the two algorithms that we set out to implement so far, namely BernsteinVazirani's Algorithm and Simon's Algorithm. Our approach is to implement as many known quantum algorithms as possible, and evolve the API as we do so. We believe that this will keep the code as simple as possible, and the solution will remain uncluttered, as classes are only added when they are required for a given algorithm. Our approach focuses on the quantum algorithms, and we attempt to make the algorithm implementations as clear as possible. This is the main idea behind the design we used so far.

Our plans for future development include implementing other known quantum algorithms such as Shor's Algorithm for factoring prime numbers, and Grover's Quantum Search Algorithm. Also, we would like to find patterns in the algorithms that we implement, and extract certain quantum circuits with generic behaviour into standalone classes, so that they can be understood and reused more easily.

Finally, we would like to create a user friendly UI on top of the API we have created in order to allow users to visually build quantum circuits, and create an XML-based file type to persist the quantum circuits to disk. The main goal is to create a framework that will help users to easily develop new quantum algorithms that solve many of the still open questions in quantum computing today.

\section{References}

1. http://www.top500.org/lists/2014/11/ (20.04.2015)

2. Gordon E. Moore, Cramming More Components onto Integrated Circuits, Electronics, vol. 38, no. 8, (1965)

3. M. Fuechsle, J. A. Miwa, S. Mahapatra, H. Ryu, S. Lee, O. Warschkow, L. C. L. Hollenberg, G. Klimeck, M. Y. Simmons, A single-atom transistor, Nature Nanotechnology, vol. 7 (2012)

4. http://www.intel.com/content/www/us/en/silicon-innovations/intel-14nm-technology.html (20.04.2015)

5. Richard P. Feynman, Quantum Mechanical Computers, Foundations of Physics, vol. 16, No. 6, (1986)

6. Lev Grossman, The Quantum Quest for a Revolutionary Computer, Time Magazine, February 17, 2014

7. IEEE Graphic Symbols for Logic Functions, IEEE Std 91a-1991 \& IEEE Std 91-1984, (1991)

8. H. M. Sheffer, A set of five independent postulates for Boolean algebras, with application to logical constants, Transactions of the American Mathematical Society, vol. 14, (1913)

9. M. Nielsen, I. Chuang, Quantum Computation and Quantum Information, Cambridge University Press (2010)

10. D. Aharonov, Wim van Dam, J. Kempe, Z. Landau, S. Lloyd, O. Regev, Adiabatic Quantum Computation is Equivalent to Standard Quantum Computation, SIAM Journal of Computing, vol. 37, (2007)

11. Umesh Vazirani, Quantum Mechanics and Quantum Computation Lecture Notes, edx.org online course, (2013)

12. http://www.quantiki.org/wiki/List_of_QC_simulators (20.04.2015).

Acknowledgment: This work was supported by the strategic grant POSDRU/159/1.5/133255, Project ID 133255 (2014), co-financed by the European Social Fund within the Sectorial Operational Program Human Resources Development 2007-2013. 